\title{
Improving intranasal delivery of neurological nanomedicine to the olfactory region using magnetophoretic guidance of microsphere carriers
}

\author{
This article was published in the following Dove Press journal: \\ International Journal of Nanomedicine \\ 10 February 2015 \\ Number of times this article has been viewed
}

Jinxiang $X^{\prime}{ }^{\prime}$

Ze Zhang'

Xiuhua $\mathrm{A} \mathrm{Si}^{2}$

'School of Engineering and Technology, Central Michigan University, Mount Pleasant, MI, ${ }^{2}$ Department of Mechanical Engineering, California Baptist University, Riverside, CA, USA
Correspondence: Jinxiang $X_{i}$ School of Engineering and Technology, Central Michigan University, 1200 South Franklin Street, Mount Pleasant, MI 48858, USA

Tel + I 9897742456

Fax + I 9897744900

Email xilj@cmich.edu
Background: Although direct nose-to-brain drug delivery has multiple advantages, its application is limited by the extremely low delivery efficiency $(<1 \%)$ to the olfactory region where drugs can enter the brain. It is crucial to developing new methods that can deliver drug particles more effectively to the olfactory region.

Materials and methods: We introduced a delivery method that used magnetophoresis to improve olfactory delivery efficiency. The performance of the proposed method was assessed numerically in an image-based human nose model. Influences of the magnet layout, magnet strength, drug-release position, and particle diameter on the olfactory dosage were examined.

Results and discussion: Results showed that particle diameter was a critical factor in controlling the motion of nasally inhaled ferromagnetic drug particles. The optimal particle size was found to be approximately $15 \mu \mathrm{m}$ for effective magnetophoretic guidance while avoiding loss of particles to the walls in the anterior nose. Olfactory delivery efficiency was shown to be sensitive to the position and strength of magnets and the release position of drug particles. The results of this study showed that clinically significant olfactory doses (up to 45\%) were feasible using the optimal combination of magnet layout, selective drug release, and microsphere-carrier diameter. A 64-fold-higher delivery of dosage was predicted in the magnetized nose compared to the control case, which did not have a magnetic field. However, the sensitivity of olfactory dosage to operating conditions and the unstable nature of magnetophoresis make controlled guidance of nasally inhaled aerosols still highly challenging.

Keywords: direct nose-brain delivery, olfactory deposition, magnetophoretic guidance, neurological nanomedicine, intranasal drug delivery, microsphere carrier

\section{Introduction}

Direct nose-to-brain drug delivery provides a noninvasive method that bypasses the blood-brain barrier and directly delivers medication to the brain and spinal cord., ${ }^{1,2}$ However, its application is limited by the extremely low delivery efficiency $(<1 \%)$ of conventional devices to the olfactory region where drugs can directly enter the brain. . $^{3,4}$ This poor bioavailability is mainly attributed to two reasons: 1) the complexity of the nasal structure that traps particles before reaching the olfactory region, ${ }^{5} 2$ ) the complete lack of control on particle motions after their release at the nostrils. The structure of a human nose is highly complex, with narrow, convoluted channels (Figure 1A). The olfactory region is located above the superior turbinate (Figure 1A), and covers about $8 \%$ of the surface area of the nasal passage. ${ }^{6}$ Due to the labyrinth structure of the nasal passage, most inhaled particles will be trapped by the nasal wall and filtered 


\section{A Nasal geometry}
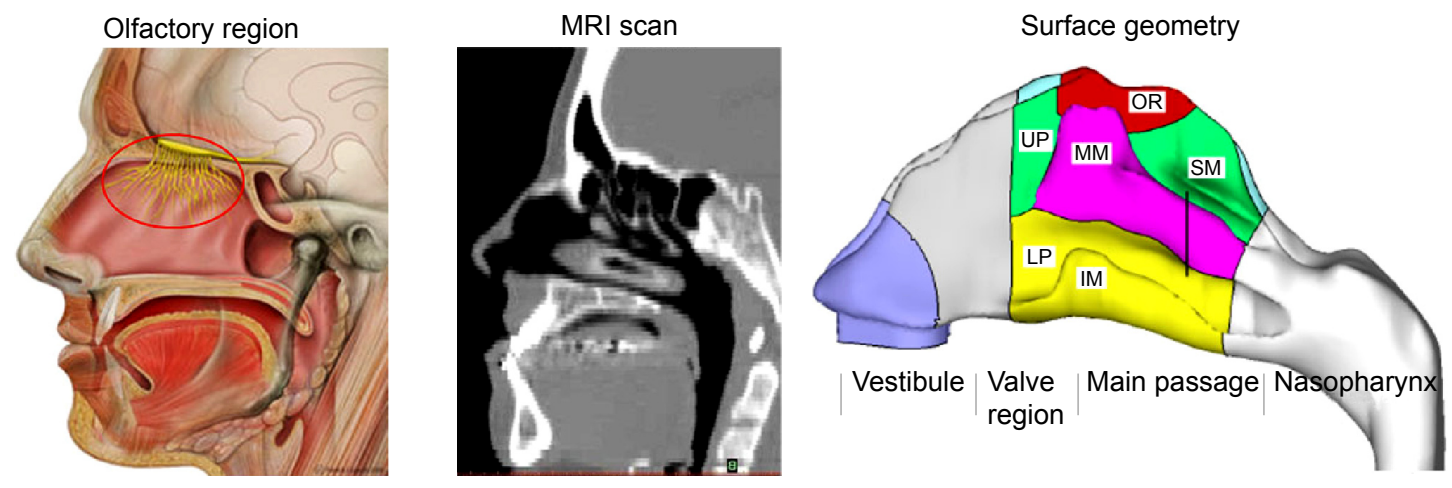

\section{B Nasal passage middle plane}
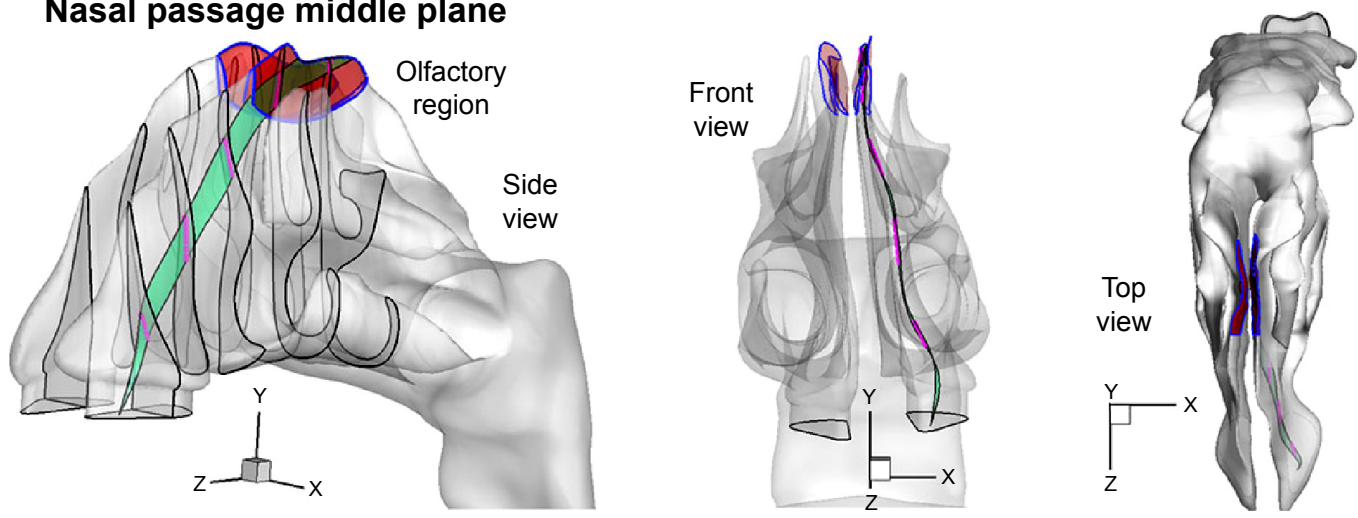

Figure I Human nose model and the olfactory region.

Notes: The red circle highlights where the olfactory region is located. (A). For optimal olfactory drug delivery, particles should travel along the middle plane (B) of the nasal passage, which exhibits complex spatial features.

Abbreviations: IM, inferior meatus; LP, lower passage; MM, middle meatus; MRI, magnetic resonance imaging; OR, olfactory region; SM, superior meatus; UP, upper passage.

out (Figure 1B). Conventional inhalation devices depend on inspiratory aerodynamics to transport therapeutic agents to the target area. ${ }^{7}$ There is no further control on the motions of particles after their release. Therefore, the transport and deposition of these particles predominantly depend on their initial velocity. Due to the convoluted nasal passage, as well as the lack of particle control, the majority of drug particles are trapped in the anterior nose and cannot reach the targeted olfactory region.

In this study, we proposed a new delivery approach that used magnetophoretic forces to enhance the olfactory delivery efficiency. A conceptual diagram of this approach is displayed in Figure 2. We hypothesized that by the application of a proper magnetic field, ferromagnetic particles can be guided through the nasal passages with a reduced loss of medication to the nasal walls compared to previous delivery methods. The magnetic force required to do so can be achieved by carefully pairing magnet strength and particle properties. Recently, Dames et al proved that it is practical to enhance drug-delivery efficacy with magnetophoresis in vivo. ${ }^{8}$ After superparamagnetic iron oxide nanoparticles (SPIONs; $80 \mathrm{~nm}$ ) had been mixed into drug aerosols, the magnetized lung received a dosage eight times higher than the control case.

Magnetic particles have been actively studied with such applications as catalysis, drug delivery, magnetic resonance or particle imaging, data storage, and optical filters. ${ }^{9}$ Magnetic drug delivery is a method used to target pharmaceuticals to a specific region in the body. Magnetic particles that are suspended in nonmagnetic fluids are subjected to positive magnetophoretic forces and are attracted toward the highest magnetic region. The major limitation in using this technique is the need for high magnetic intensity and high magnet gradient, both of which are necessary for effective control. Stationary magnets of safe strengths can only focus particles about $5 \mathrm{~cm}$ deep under the skin. Attempts are also underway to target deep tissue with dynamic magnetic control, up to $20 \mathrm{~cm}$ away from the magnet surface. ${ }^{10}$ However, until such a system is available, the magnet has to be placed close to the diseased region. At the moment, 


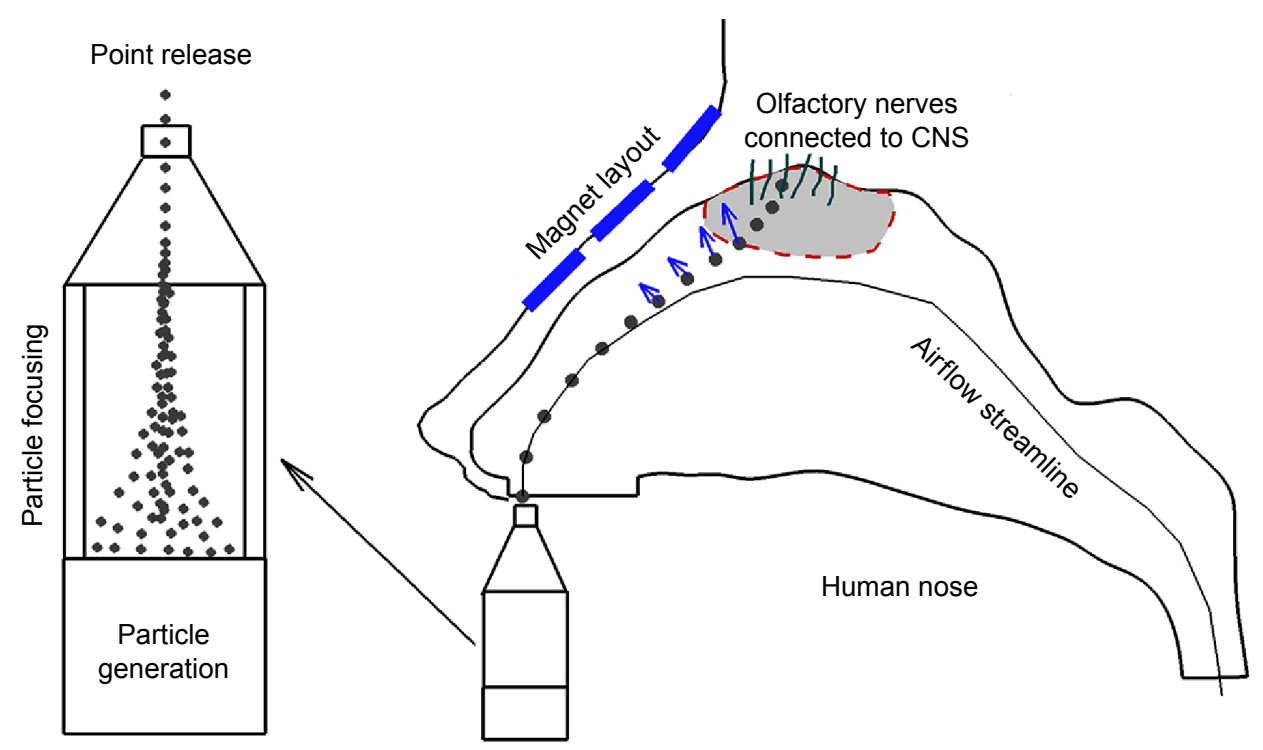

Figure 2 Diagram of magnetophoretically guided drug delivery.

Notes: By the application of an appropriate magnet layout, particles can be guided through the geometry of the nasal passage with reduced contact with the wall. Point release of drug particles is recommended for optimal delivery efficiency.

Abbreviation: CNS, central nervous system.

pathologies in or close to the skin are the major candidates for magnetic drug delivery. Examples that can be targeted with magnetic drug delivery are the skin, breast, liver, prostate, colon, and brain. ${ }^{11}$

Most of the previous studies of magnetic drug delivery concerned liquids (eg, blood flow), mucous, soft tissue, or skin, where the magnetic agents could suspend relatively long and evenly. ${ }^{12}$ This suspension cannot be readily realized in low-density fluids, such as air, for magnetic microspheres. Furthermore, the particle inertia relative to the viscous drag is much larger in airflow than in liquids (eg, the Reynolds number in the air is about 55 times greater than that of water at room temperature). In order to effectively guide the intranasally delivered drugs to the olfactory region, it is necessary for the applied magnetophoretic forces to overcome both the particle inertia and gravitational force. Experimental studies exploring magnetophoretic control for applications in inhalation drug delivery include 1) Brain et al who used iron oxide particles to improve the deposition in rabbit lungs, ${ }^{13}$ 2) Stahlhofen and Möller who investigated the dynamics of magnetic microparticles in human lungs, ${ }^{14} 3$ ) Jang and Cao, who measured the deposition fraction of magnetic nanoparticles, ${ }^{15}$ and 4) Martin and Finlay, who managed to enhance fiber depositions using magnetic alignment. ${ }^{16}$ More recently, Xie et al conducted a numerical study of magnetic aerosols in the range of $0.25-4.5 \mu \mathrm{m}$, and demonstrated that numerical predictions could adequately describe the complementary in vitro deposition experiments. ${ }^{17}$
There were three objectives in this study: 1) assess the feasibility of magnetophoresis to improve the olfactory dosage of nasally inhaled particles, 2) identify the strength of magnets and the sizes of drug carrier that are necessary for meaningful olfactory dosages, and 3) identify the synthesis of the magnetic and particle parameters to achieve optimal olfactory delivery.

\section{Materials and methods Magnetophoretic olfactory delivery protocol}

The proposed device consists of two rows of permanent magnets positioned above the nose. In each row, there are multiple magnet bars, each having different strengths to produce a specific magnetic field within the nasal cavity. Once released into the nose, a drug particle will experience magnetic attractions and depart from its original course. With an appropriate magnetic field, drug particles can be guided to the olfactory region with reduced losses to nasal walls. Permanent magnets and ferromagnetic drug particles were tested in this study, because this combination is accepted as the simplest and most cost-effective manipulation method among magnetic controls. ${ }^{18}$

\section{Fluid-particle transport models}

The inhaled airflows were assumed to be steady, incompressible, and isothermal in this study. A laminar flow model was adopted to resolve the flow field. ${ }^{19-21}$ A Lagrangian tracking 
approach was implemented to simulate the trajectories of particles. $^{22}$ The particles were assumed to be spherical, and ranged from $1 \mu \mathrm{m}$ to $30 \mu \mathrm{m}$ in diameter. The governing equation is: ${ }^{23}$

$$
\begin{aligned}
\frac{d\left(m_{p} v_{i}\right)}{d t}= & \frac{f}{\tau_{p} C_{c}} m_{p}\left(u_{i}-v_{i}\right)+m_{p} g_{i}(1-\alpha) \\
& +F_{i, \text { Lift }}+F_{i, \text { Magnetophoretic }}
\end{aligned}
$$

where $v_{i}$ and $u_{i}$ are particle velocity and fluid velocity, respectively. The symbol $C_{c}$ is the Cunningham slip-correction factor, $\tau_{p}\left(\mathrm{ie}, \rho_{p} d_{p}^{2} / 18 \mu\right.$ ) is the characteristic time for a particle to respond to changes in the airflow, $\rho_{p}$ is the particle density, and $d_{p}$ is the particle diameter. The drag factor $(f)$ is calculated following the equation suggested by Morsi and Alexander. ${ }^{24}$ For micrometer particles, the Saffman lift force was also included. ${ }^{5}$ Diluted particle concentrations were assumed in this study.

\section{Magnetic field and magnetophoretic force}

The relation between magnetic flux density $(B)$, magnetic intensity $(H)$, and magnetization $(M)$ is given by:

$$
B=\mu_{0}(H+M)
$$

where $\mu_{0}$ is the permeability of free space. $M$ includes permanent magnetization and any linear and nonlinear function of $H .{ }^{25}$ We assume that there is no electric current flow: $\nabla \times H=0$. The effective magnetic dipole moment (ie, magnetization) induced by the magnetic field $(H)$ is given by:

$$
m_{\text {eff }}=\frac{4}{3} \pi d_{p}^{3} K H
$$

where $K$ is the Clausius-Mossotti factor:

$$
K=\frac{\mu_{p}-\mu_{f}}{\mu_{p}+2 \mu_{f}}
$$

The magnetophoretic force acting on a spherical particle in a nonuniform magnetic field is computed by: ${ }^{26}$

$$
F_{i, \text { Magnetophoretic }}=\left(\mu_{0} m_{\text {eff }} \cdot \nabla\right) H
$$

The symbols $\mu_{p}$ and $\mu_{f}$ are the magnetic permeability of particles and fluid, respectively. From the Equation 5, it is apparent that the magnetophoretic force is proportional to particle volume, the strength of the magnetic field, and the magnetic field gradient. In addition, the force is directed along the gradient of the external magnetic field.
The particle dynamics relative to applied magnetic fields were first studied in a simple two-plate channel. For given particle-release positions and inhalation conditions, the required field strengths for effective magnetic manipulation were computed. These computed parameters were subsequently used in both idealized and realistic nose models to assess the olfactory delivery efficiencies. The use of an idealized nose model could remarkably reduce the requirement of computational times relative to the realistic nose model. Influences of operating parameters, such as the strength and location of external magnets, drug-release positions, and particle size, on the olfactory delivery efficiency were investigated.

\section{Numerical methods}

The nose model implemented in this study was based on magnetic resonance scans of a 53-year-old male. Detailed procedures to construct this model had been reported in $\mathrm{Xi}$ and Longest. ${ }^{19}$ Multiphysics ${ }^{\circledR}$ software (Comsol, Burlington, MA, USA) was used to solve the airflow, magnetic field, and particle tracing. A mesh-independence test was undertaken by comparing predicted aerosol depositions from various mesh densities, which ranged from 0.4 to 2.0 million computational cells. The final mesh for numerical analysis in this study consisted of 1.1 million cells.

\section{Results}

\section{Magnetophoretic control in a two-plate channel}

The feasibility of magnetophoretic control of particle motions was first assessed in a two-plate channel (Figure 3) with various magnet layouts. By setting multiple permanent magnets on the outside edges of the two plates, the trajectory of particles could be modified within the channel. In this simulation, the length of the channel was $150 \mathrm{~mm}$, and the height was $10 \mathrm{~mm}$. The magnetic permeability of the particles was $880 \mu \mathrm{H} / \mathrm{m}$, and the relative magnetic permeability was $700 \mu \mathrm{H} / \mathrm{m}$. The particle size tested was in the range of $1-30 \mu \mathrm{m}$. The density of particles was $1,500 \mathrm{~kg} / \mathrm{m}^{3}$ considering that the droplets were a mixture of water and iron nanoparticles. The airflow and particles were inhaled into the nostrils at $0.5 \mathrm{~m} / \mathrm{s}$. The magnetic field and associated particle dynamics within the two plates are presented in Figure 3 for three different magnet settings.

Because magnetophoretic force is proportional to the particle volume $\left(d_{p}{ }^{3}\right.$, Equation 3$)$, while the viscous drag force is proportional to the particle diameter, it is more difficult to control smaller particles. For a given magnetization of $1 \times 10^{5} \mathrm{~A} / \mathrm{m}$, only particles larger than $10 \mu \mathrm{m}$ show noticeable magnetic responses. Figure $3 \mathrm{~A}$ shows the particle 
A Without magnetophoresis (control case)

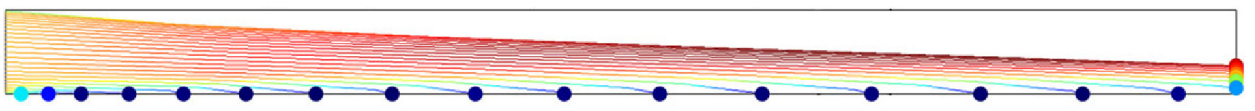

B Magnet layout 1

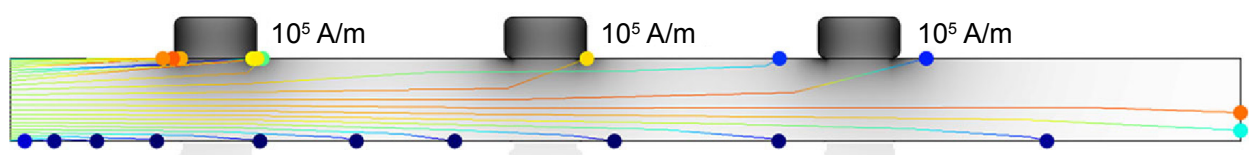

C Magnet layout 2

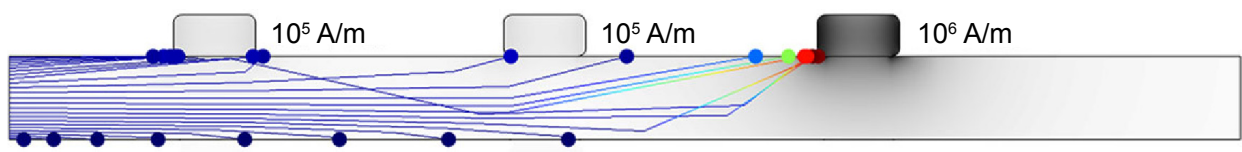

D Magnet layout 3

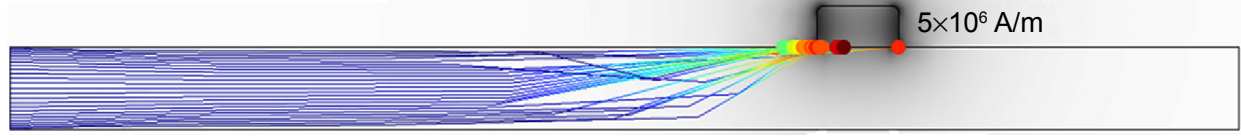

Figure 3 Magnetic field and particle trajectories within a two-plate channel.

Notes: (A) control case; (B) layout I; (C) layout 2; (D) layout 3.

trajectories of $15 \mu \mathrm{m}$ with no magnetic field (control case), where particles follow parabolic path lines due to the gravitational force. As expected, a particle with a release position that was closer to the bottom plate settled earlier (due to short ambient time), while a particle with a release position closer to the upper plate settled after traveling a longer distance. In order to manipulate the particle motions, the applied magnetophoretic force must be sufficient to overcome the effects of gravity and particle inertia.

In the first trial, we tested the feasibility of controlling particle motions by using magnetophoretic forces to counteract gravity, allowing the particles to move horizontally instead of falling. To this purpose, we applied three magnets on top of the channel (Figure 3B). The resultant magnet field was nonuniform, being stronger at the upper plate and weaker at the bottom plate. The ferromagnetic particles were attracted upward toward the stronger magnetic field, which acted against gravity. When all three magnets had a volume magnetization of $1 \times 10^{5} \mathrm{~A} / \mathrm{m}$ and the given particle size was $15 \mu \mathrm{m}$, the magnetophoretic force was in equilibrium with the gravitational force at the centerline of the channel (Figure 3B). However, this force had a linear dependence on the strength of the magnetic field. The upper part of the channel had a stronger magnetophoretic force, which pulled particles toward the top plate. On the other hand, the particles in the lower channel continued along their original path, because the magnetophoretic force was weaker than gravity. It was also noted that compared with the trajectory in the control case (no magnetic field), the particles fell at a much slower rate, due to the upward magnetophoretic force.

The second trial tested how the particle trajectories changed when stronger magnets were applied (Figure 3C). In this trial, the left two magnets were kept at $1 \times 10^{5} \mathrm{~A} / \mathrm{m}$, while the right magnet was increased to $1 \times 10^{6} \mathrm{~A} / \mathrm{m}$. Since the magnetic field was much stronger at the right side, all particles that passed through the left half of the channel turned their direction upward and deposited in the area surrounding the third magnet. This trial demonstrated that when the magnetophoretic force was strong enough, the particle motion could be manipulated to reach the desired area. With an even stronger magnet (eg, $5 \times 10^{6} \mathrm{~A} / \mathrm{m}$ ), nearly all particles were attracted to it, as illustrated in Figure 3D. These results show promising enhancement of the delivery dose to the nasal olfactory region by using magnetophoretic guidance.

\section{Two-dimensional nose geometry}

The performance of the magnetophoretic guidance was further assessed in an idealized 2-D nose model. The airflow field within the nose is shown in Figure 4A. Only a small portion of the inhaled airflow is conveyed to the upper nasal cavity, indicating a low probability of particle transport to this region. From Figure 4A and B, streamlines that initiate from 


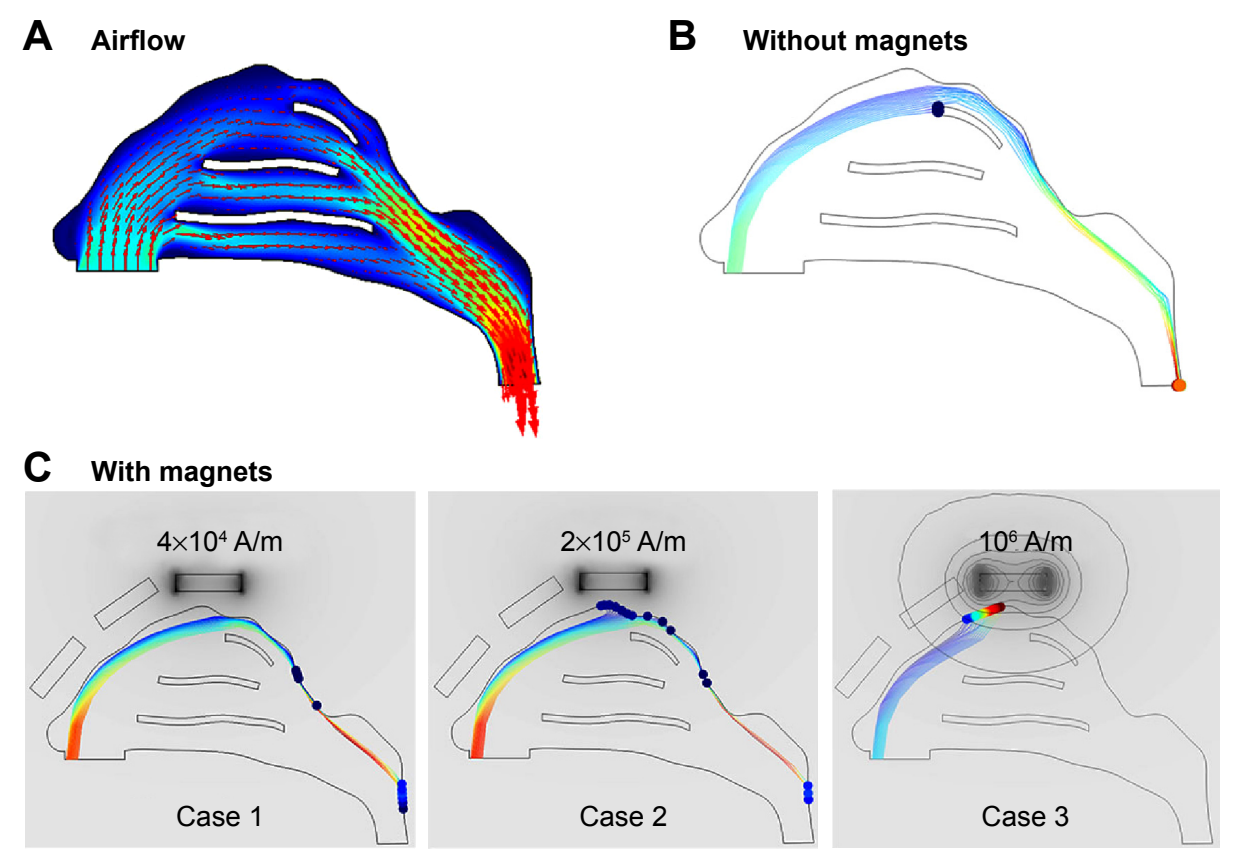

Figure 4 Airflow (A) and particle trajectories in the 2-D nose without magnets (B) and with magnets (C). Note: Darker color in (C) represents a stronger magnetic field.

the nostril tip travel toward the upper nasal passage and those from the nostril base travel toward the inferior nasal passage. As a result, particles that are released from the nostril tip will be more likely to travel to the olfactory vicinity.

The magnet layout in the 2-D nose model consisted of three permanent magnets that were placed close to the nose. The size of the magnets was $5 \times 20 \mathrm{~mm}$. The first two magnets had zero-volume magnetizations. For the third magnet, which was above the olfactory region, three volume magnetizations were tested, with case 1 being $4 \times 10^{4} \mathrm{~A} / \mathrm{m}$, case $22 \times 10^{5} \mathrm{~A} / \mathrm{m}$, and case $31 \times 10^{6} \mathrm{~A} / \mathrm{m}$ (Figure $4 \mathrm{C}$ ). The resulting magnetic field is shown in Figure $4 \mathrm{C}$ in terms of both contour and darkness of the gray color. The strength of the magnetic field was observed to decrease quickly with increasing distance from the permanent magnet, as evidenced by the contrast between the deep-gray color near the magnet and the lightgray color 3-5 mm from the magnet (Figure 4C). Similarly, the magnetic gradient also dissipated quickly, as indicated by the highly condensed contour near the magnet versus the sparsely distributed contours away from the magnet. Considering that the magnetophoretic force is proportional to the product of the intensity and gradient of the magnetic field (Equation 5), its impact would decrease even more quickly (with an exponent of 2) as it moves away from the magnet.

In our previous studies, it had been shown that selective particle release could led to enhanced olfactory dosages compared to releasing drugs into the entire nostril., ${ }^{4,27}$ To reduce unwanted drug waste in the nose, particles were only released from the anterior nostril. Figure $4 \mathrm{C}$ shows the particle transport and deposition after releasing the particles from one point at the tip of the nostril with a different magnet layout. It is shown that particle trajectories deviate upward, due to the presence of magnets above the nose (Figure 4C). Furthermore, with appropriate magnet strength $\left(1 \times 10^{6} \mathrm{~A} / \mathrm{m}\right.$ in case 3$)$, the majority of magnetophoretic-driven particles from this point $(\sim 92 \%)$ deposit in the olfactory region. By contrast, an inadequate magnet field yields less pronounced magnetic responsiveness (cases 1 and 2). In the absence of magnets, nearly no particles deposit in the olfactory region, even though the particles will pass by the olfactory region (Figure 4B).

Figure 5 shows the olfactory dosages for $15 \mu \mathrm{m}$ particles at both logarithmic and linear scales. For the given magnetic layouts, as shown in Figure 4, particles smaller than $15 \mu \mathrm{m}$ cannot be drawn upward, and those larger than $15 \mu \mathrm{m}$ will be more likely to deposit in the nasal valve region. As observed in Figure 5, applying magnetophoretic forces significantly increases the olfactory dosage. The magnetized olfactory dosages are more than one order of magnitude higher than those without magnetophoretic control. In addition, releasing particles from a selective point at the nostril (point release) results in more effective performance than releasing particles from the entire nostril (Figure 5).

\section{Three-dimensional realistic nose model}

Figure 6A shows the airflow fields and particle trajectories of $15 \mu \mathrm{m}$ particles in the 3-D nose model at an 

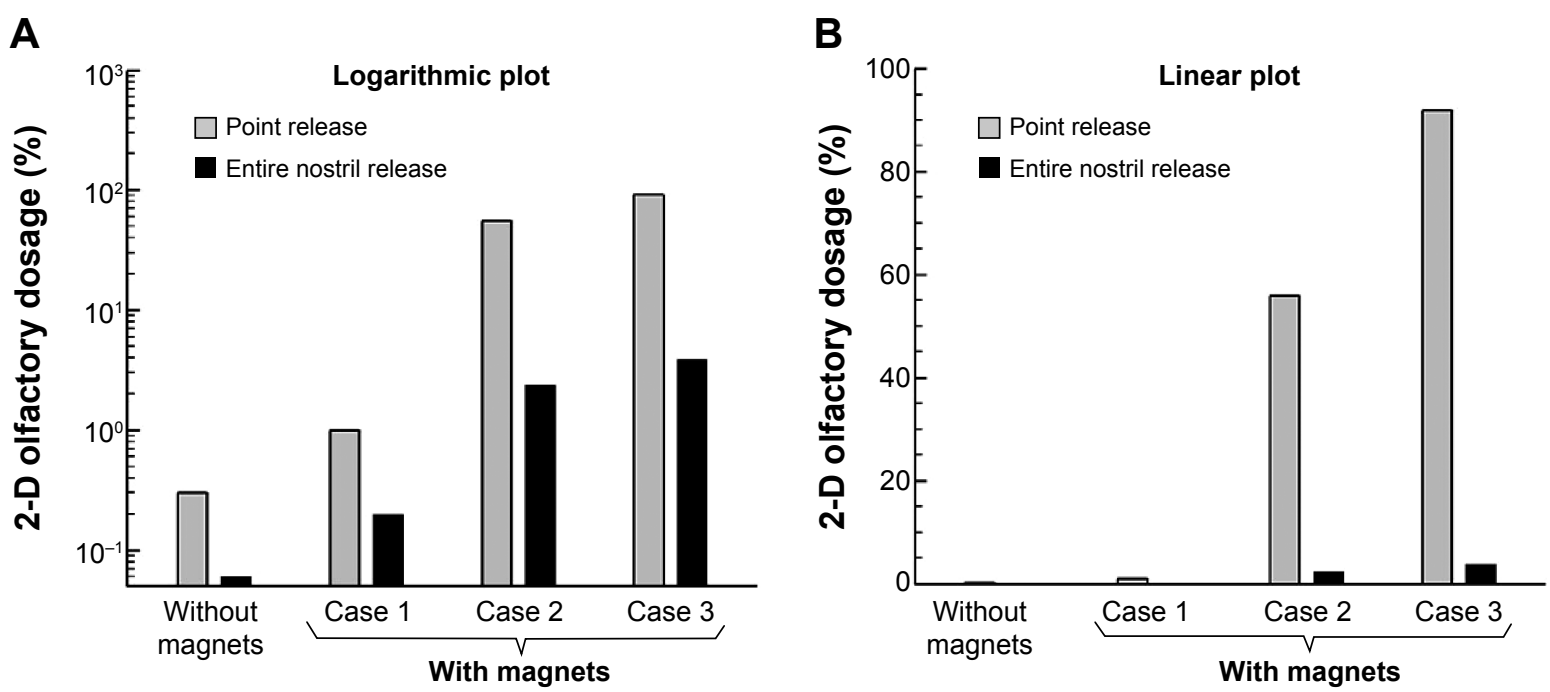

Figure 5 Comparison of olfactory dosages among different magnet layouts in the 2-D model at (A) logarithmic scale and (B) linear scale.

inhalation velocity of $0.5 \mathrm{~m} / \mathrm{s}$. The inhaled airflow moves faster in the medial passage and slower in the fin-like meatus regions. Only streamlines seeded from the nostril tip reach the olfactory region. Without magnetophoretic guidance, a negligible fraction of particles deposits in the upper passage; even fewer particles deposit in the olfactory receptor (Figure 6A).

Two designs of the magnet layouts (layouts $\mathrm{A}$ and $\mathrm{B}$ ) are shown in Figure 6B and C, respectively. Layout A is the baseline design, while layout $\mathrm{B}$ is an improved design.

A Without magnet
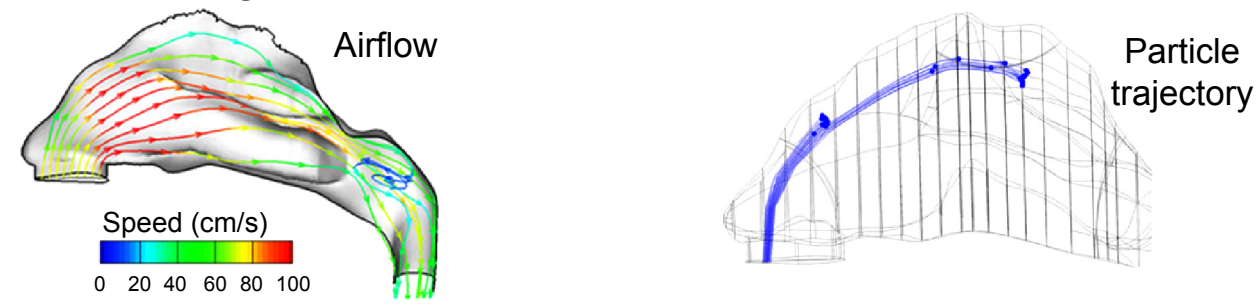

B Magnet layout A
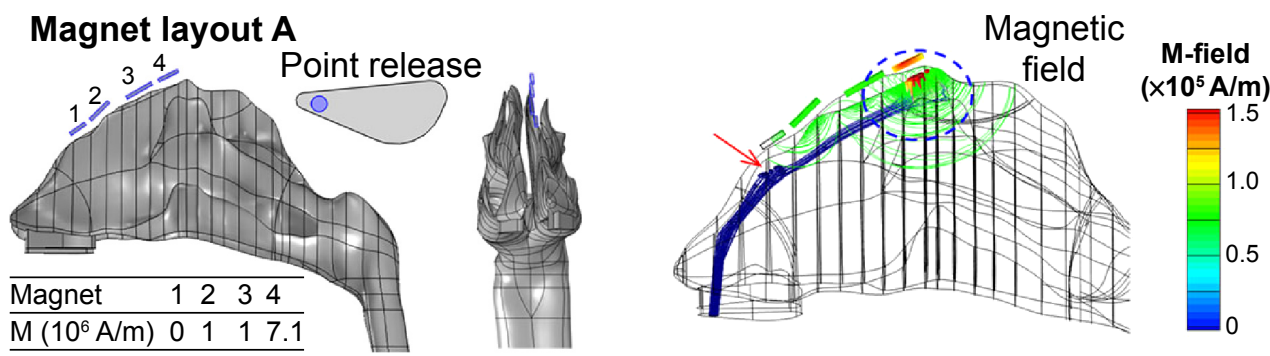

\section{Magnet layout B}
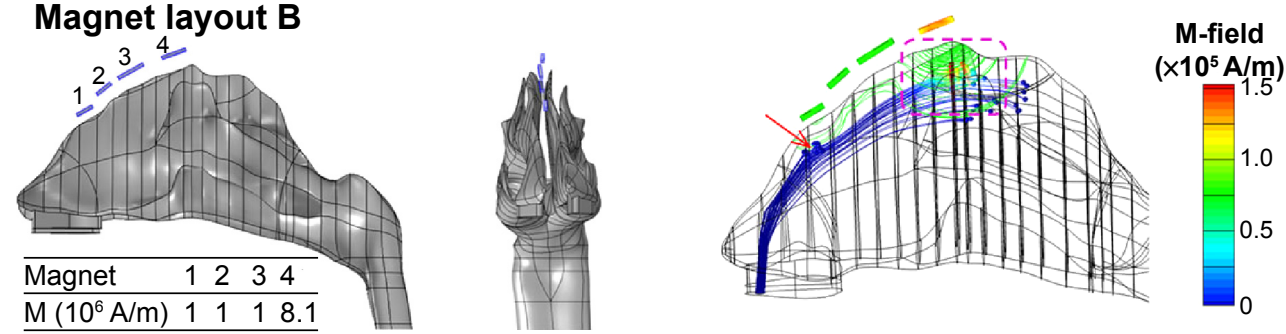

Figure 6 Comparison of particle motions in an image-based 3-D nose model among different magnet layouts.

Notes: The airflow and particle trajectories without magnets are shown in (A). The magnet layout and strength of two protocols are shown in (B) layout A and (C) layout B. Their magnetic fields and particle trajectories are also shown.

Abbreviations: M, magnetization, M-field, magnetic field. 
Table I Volume magnetization $(M)$ of the magnet bars

\begin{tabular}{lllll}
\hline $\mathbf{M}\left(10^{5} \mathbf{A} / \mathbf{m}\right)$ & $\mathbf{I}$ & $\mathbf{2}$ & $\mathbf{3}$ & $\mathbf{4}$ \\
\hline Layout A & 0 & $\mathrm{I}$ & $\mathrm{I}$ & 7.1 \\
Layout B & $\mathrm{I}$ & $\mathrm{I}$ & $\mathrm{I}$ & 8.1 \\
\hline
\end{tabular}

With regard to layout A (Figure 6B), one row of magnets are positioned along the ridge of the left nasal passage (Table 1), which generate a magnetic field that is strong in the olfactory region and weak in other nasal regions. In this study, particles are released from a $1.5 \mathrm{~mm}$-diameter nozzle (point release) into the left nostril (Figure 6B). These particles are assumed to be ferromagnetic, with a relative permeability of 700 , uniform in diameter, and have a density of $1.5 \mathrm{~g} / \mathrm{cm}^{3}$.

A comparison of particle trajectories with and without magnetophoretic guidance is shown in Figure 6B versus A. In the absence of magnetophoretic guidance, most particles deposited on the nasal valve and side walls of the nasal turbinates, as illustrated in Figure 6A. For those particles that passed through the upper nasal cavity, the downward convective drag force prevented them from reaching the olfactory region. To remedy this, one row of magnets was applied on the top of the nasal airway. In accordance with the parameters obtained in the 2-D nose model, magnets with a volume magnetization of $1 \times 10^{6} \mathrm{~A} / \mathrm{m}$ were employed. However, the olfactory delivery in this trial did not show promising results, presumably due to inadequate upward magnetophoretic force to reverse the particle motion. This inadequacy could be attributed to the unique airflow and pressure distributions in the realistic 3-D nose model, which were far more complex than those in the idealized 2-D nose model. The predicted discrepancy between the 2-D and 3-D models also suggested that a physiologically realistic model was indispensable in order to develop clinically relevant protocols.

To identify the appropriate magnet strength for effective olfactory delivery, a variety of volume magnetizations were tested by progressively increasing magnetization from $1 \times 10^{6} \mathrm{~A} / \mathrm{m}$ by an increment of $1 \times 10^{5} \mathrm{~A} / \mathrm{m}$. It was observed that after an increase in maximum magnetization to $7.1 \times 10^{7} \mathrm{~A} / \mathrm{m}$ (detailed magnet strength listed in Figure $6 \mathrm{~B}$ ), about $33 \%$ of the administered particles reached and deposited in the olfactory region (Figures $6 \mathrm{~B}$ and $7 \mathrm{~B}$ ). About $48 \%$ of the released particles deposited around the nasal valve, which should be avoided ideally.

To further enhance olfactory dosages, we modified the magnet layout to minimize drug losses to the nasal valve and to the outside of the olfactory region. A careful inspection of the nasal valve deposition (Figure 6B) revealed that particles were deposited on the outer wall (left); therefore, by applying a lateral force to the right, the deposition loss could be reduced. Similarly, the depositions anterior to the olfactory boundary were caused by a strong, upward magnetic force, which caused an early particle deposition. This loss could be reduced by either decreasing the magnet strength or moving the magnet further away from the nose (Figure 6C, layout B). After extensive testing, an optimized delivery protocol was identified, as shown in Figure 7C. In this optimized case, about $45 \%$ of therapeutic agents deposited in the olfactory region, while drug losses to the nasal valve and to the outside of the olfactory boundary were remarkably reduced. This is illustrated by the red arrow (Figure 7A and B versus $\mathrm{C}$ ) and purple circle (Figure 7B versus C), respectively.

Figure 7D shows the time evolution of olfactory deposition for the three magnet layouts. For the given inhalation rate $(0.5 \mathrm{~m} / \mathrm{s})$ and particle size $(15 \mu \mathrm{m})$, it took about 0.1 seconds for the particles to first reach the olfactory region. However, due to the highly heterogeneous flow field, it took another 0.2 seconds for the remaining particles to deposit in the olfactory receptor. When layouts A and B are compared, moving magnets further from the nose led to a more desirable deposition pattern (Figure 7A versus B), but also reduced the total olfactory dosage by $25 \%$ (Figure 7D). This reduced dosage was mainly caused by a decreased magnetophoretic force; therefore, fewer particles were drawn to the olfactory region. Noticing that such particles were from certain regions of the release orifice, we refined the shape and size of the orifice (layout $\mathrm{C}$ in Figure 7C), which substantially reduced unwanted depositions and enhanced the olfactory dosage.

The benefits of point release of drugs are demonstrated in Figure 8 by its much-elevated delivery efficiency ( $45 \%)$ than releasing drugs into the entire nostril, which has very low delivery efficiency (1.205\%) even with magnetophoretic guidance. Due to the high inertia of $15 \mu \mathrm{m}$ droplets, drugs released from the whole nostril will mostly deposit in the anterior nose. Therefore, point release should be implemented to deliver clinically significant dosages to the olfactory receptor.

The predicted olfactory dosages of $15 \mu \mathrm{m}$ aerosols with and without magnetophoretic guidance are shown in Figure 9A and B, at logarithmic and linear scales, respectively. The predicted depositions in the realistic 3 -D model are consistent with the 2-D results, such that magnetophoretic guidance significantly improves olfactory dosages, and that point release is superior to conventional release from the entire nostril. With appropriate magnetophoretic guidance, the delivered olfactory dose can be one or even two orders of magnitude higher than that 


\section{A Layout A}

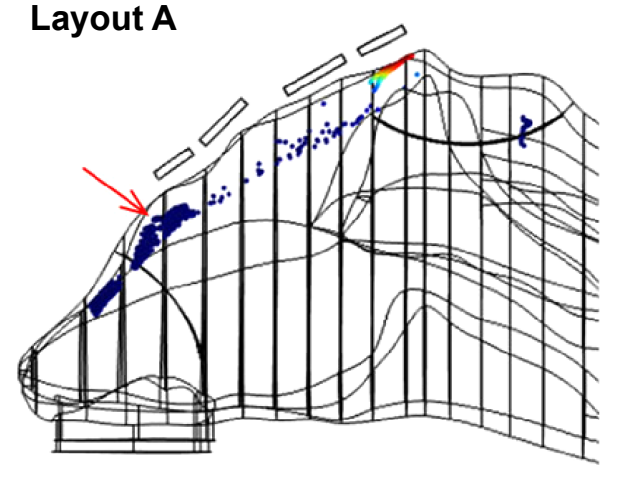

\section{C}
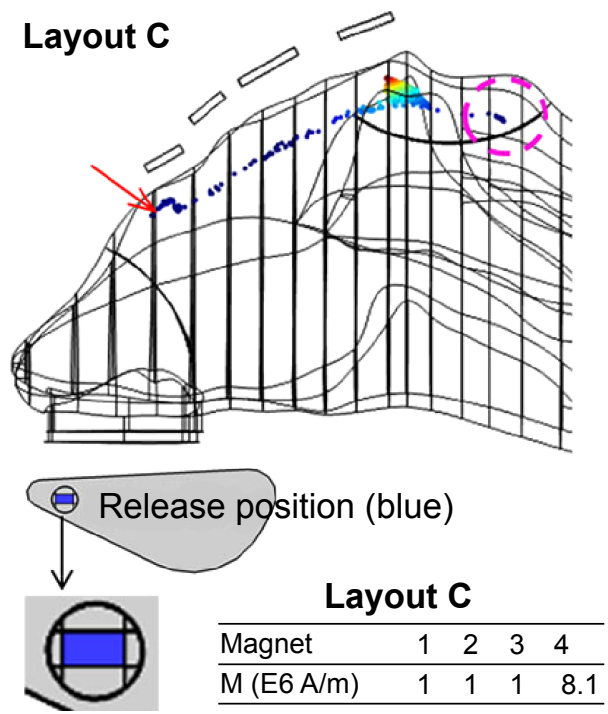

B

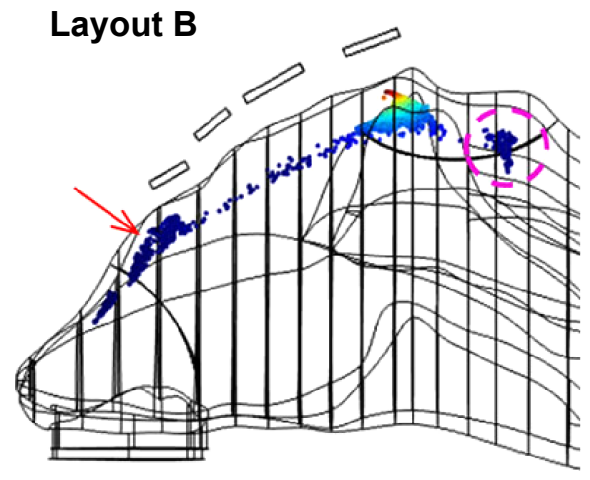

Olfactory deposition

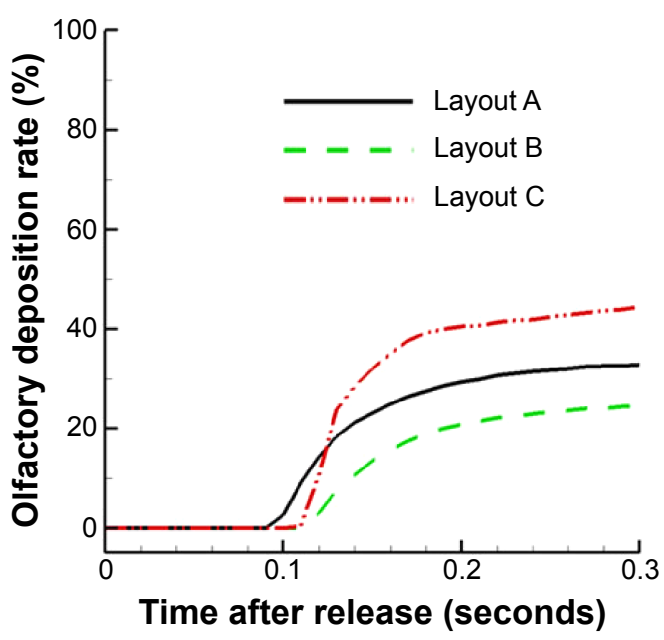

Figure 7 Comparison of surface depositions among various magnet layouts.

Notes: (A) Layout A; (B) layout B; (C) layout $C$. The only difference between layouts B and C is that the latter has a smaller release area. The time evolution of olfactory deposition for the three magnet layouts is shown in (D). Red arrow indicate the nasal valve.

Abbreviation: $M$, magnetization.
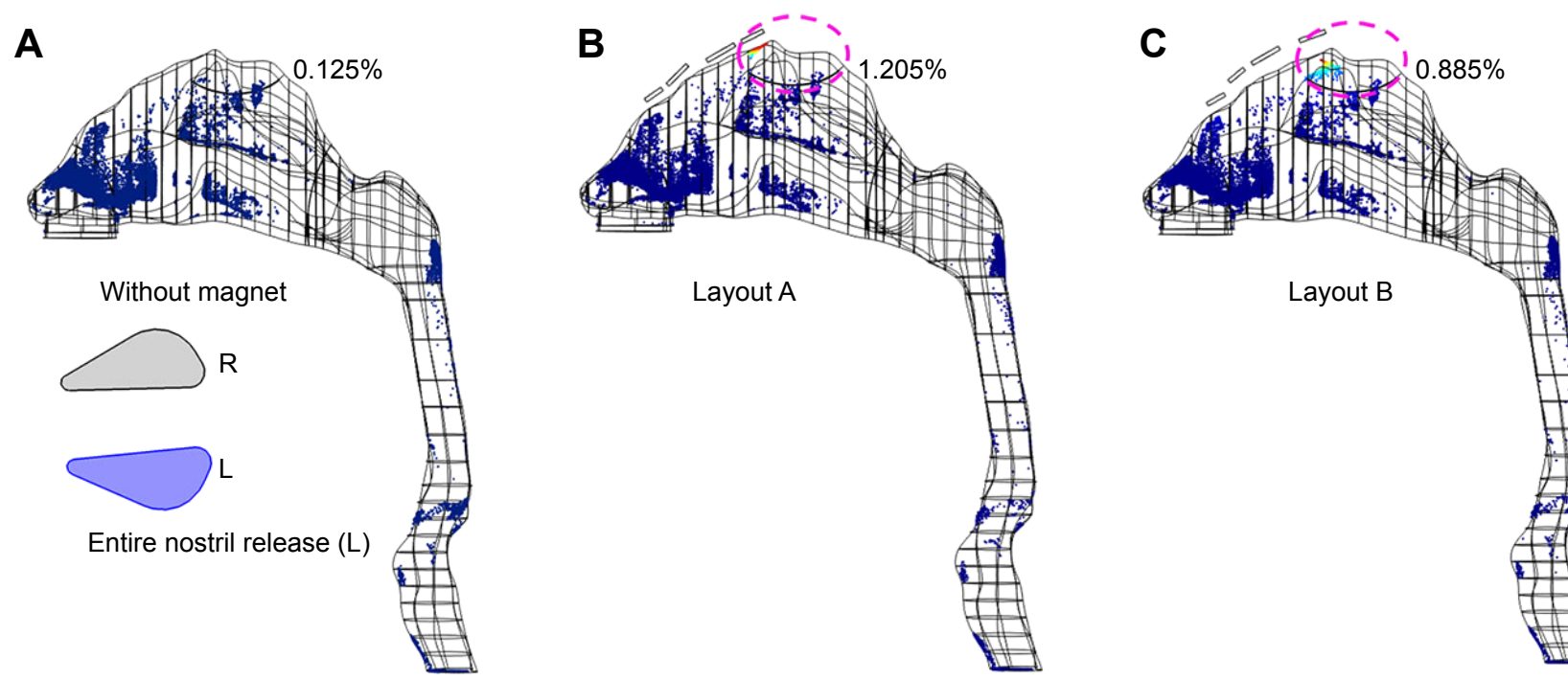

Layout $A$

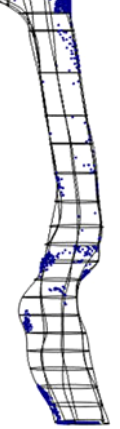

Layout B

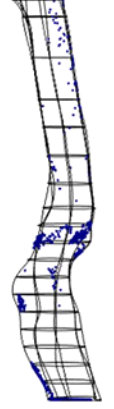

Figure 8 Surface deposition with entire-nostril release.

Notes: Benefits of point release of drugs are demonstrated by its superiority to releasing drugs into the entire nostril, which delivers insignificant dosage to the olfactory region even with magnetophoretic guidance (1.205\%). Pink circle indicates the olfactory region.

Abbreviations: L, left; R, right. 

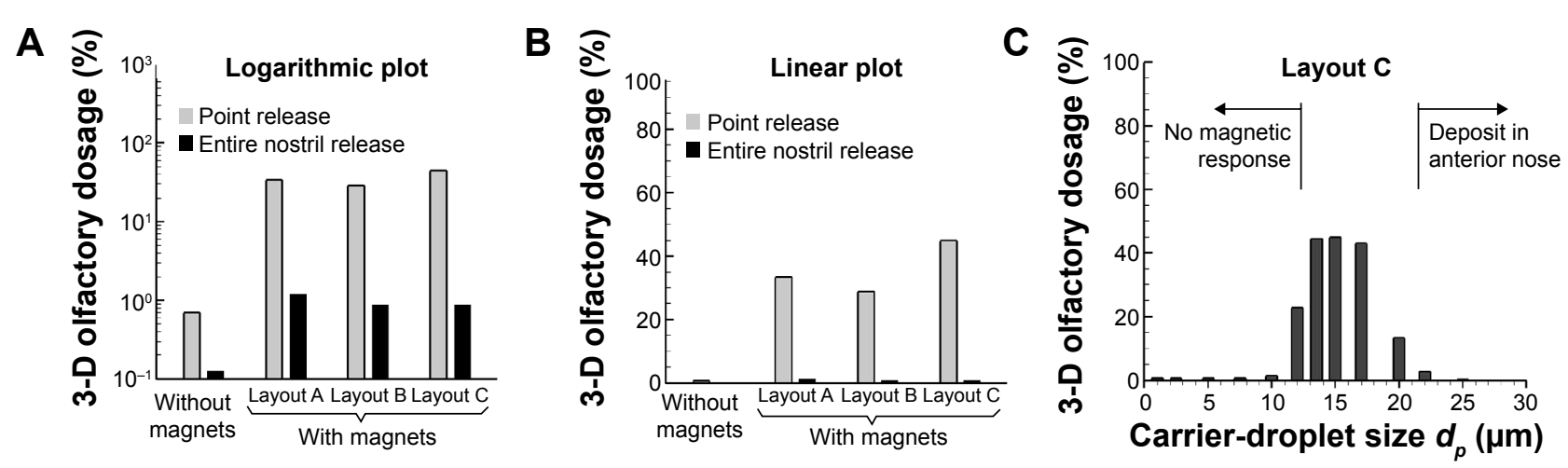

Figure 9 Olfactory dosages in the realistic 3-D nose model.

Notes: Comparison of olfactory dosages among various magnet layouts is shown at (A) logarithmic scale and (B) linear scale. The variation of 3-D olfactory dosages versus carrier-droplet size is shown in (C).

without magnetophoretic guidance (Figure 9A). However, lower olfactory dosages were predicted in the realistic 3 -D model (45\%, Figure 9B) compared to that of the 2-D model ( $>90 \%$, Figure 5B). This finding was not surprising, because the olfactory dosage had been noted to be highly sensitive to geometrical details and operating variables (eg, magnetization). ${ }^{4}$

The variation of the 3-D olfactory dosage as a function of carrier-droplet size is shown in Figure 9C. The magnet configuration implemented is layout $\mathrm{C}$ (the optimal design in this study) and the aerosol size $\left(d_{p}\right)$ ranges from 1 to $30 \mu \mathrm{m}$. From Figure 9C, there is negligible olfactory deposition for $d_{p}<10 \mu \mathrm{m}$ or $d_{p}>20 \mu \mathrm{m}$; the former is due to weak magnetic responsiveness, while the latter is due to the high inertia loss to the anterior nose. The optimal olfactory deposition comes from aerosols in the range of $13-17 \mu \mathrm{m}$, with a median size of $15 \mu \mathrm{m}$.

\section{Discussion and summary}

Results of this study show that with a proper magnetic field, significantly improved olfactory delivery can be achieved by guiding nasally inhaled particles toward the olfactory region. In this study, it was demonstrated that with magnets of strength of $8 \times 10^{6} \mathrm{~A} / \mathrm{m}$ outside the nose, the optimal particle size should be around $15 \mu \mathrm{m}$, in order to effectively steer drug particles to the olfactory region. Smaller particles will not change their path, due to small magnetophoretic forces, while larger particles will most likely deposit in the anterior nose, due to large inertial impaction. Results in the 3-D realistic nose model showed significantly enhanced olfactory dosage (45\%) with magnetophoretic guidance compared to that without guidance $(0.7 \%)$. Also, releasing drug particles from selected points instead of the entire nostril can further reduce drug waste. The location of the magnets will also noticeably affect particle transport and deposition.

A major limitation of drug delivery with permanent magnets and ferromagnetic particles lies within the magnetophoretic force on the particle, which depends on the magnetic moment of the particle and the gradient of the magnetic field. Magnetic fields of safe ranges are only strong enough to control particle motions a few millimeters from the edge of a magnet, which fortunately is still applicable for intranasal drug delivery. ${ }^{28}$ Theoretically, there are two options to increase the magnetic response of the particles: enhance the magnetic moment of the particles, or increase the magnetic field gradients. The magnetic moment of a particle depends on both its material and size. Using larger particles will increase the magnetic response, but will also increase the chance of wall loss, due to larger inertial impaction. There is a chance of increased rates of agglomeration for ferromagnetic particles. ${ }^{29}$ However, this effect can be advantageous in this application, as it increases the magnetophoretic force.

The diameter of the carrier droplet is also a critical parameter for effective olfactory deliveries. Larger droplet diameter will provide greater magnetic responsiveness. Dames et al theoretically showed that for a single SPION $(80 \mathrm{~nm})$, the magnetophoretic force was not large enough to control its motions. ${ }^{8}$ However, when SPIONs were assembled into a microsphere droplet or particle, they became guidable, due to an increased size and associated magnetophoretic responsiveness. The optimal diameter of the carrier particle was found to be around $15 \mu \mathrm{m}$ for the given magnets. In practice, this size can be readily generated from a liquid suspension containing SPIONs. Our numerical experiments in this study showed that clinically significant olfactory doses (up to $45 \%$ ) were feasible using the optimal combination of magnet layout, selective 
drug release, and carrier-droplet diameter. A 64-fold-higher dose was predicted in the magnetized nose compared to the control case $(0.7 \%)$, which was without a magnetic field.

The major challenge with magnetophoretic-guided drug delivery is the quick decay of the magnetic field away from the magnet. For instance, the magnetic intensity decreases by $90 \%$ at a distance of $5 \mathrm{~mm}$ from the magnet. There are several alternatives to improve the magnetic manipulation in this study. Elongated particles have a larger magnetic moment compared to spherical particles, and therefore could be more suitable for magnetic drug delivery. ${ }^{30}$ A charged particle moving in a magnetic field will experience acceleration normal to its motion, which can be used to manipulate particle motion in regions of weak magnetic gradients. Furthermore, dynamic magnetic control can be used to achieve deeper drug-target delivery, due to the large, ever-changing magnetic gradients. ${ }^{10}$ Still another alternative is to use nonmagnetic drug particles in a ferrofluid (eg, airflow with a colloidal suspension of superparamagnetic nanoparticles). In this case, the nonmagnetic particles in a nonuniform magnetic field will experience repulsive forces; hence, they will be trapped in the minima regions of magnetic intensity. ${ }^{26,31}$

In summary, performances of the proposed magnetophoretic guidance were numerically assessed using both 2-D and 3-D nose models. Design parameters, such as magnet location, drug-release position, and particle size, were examined regarding their impacts on olfactory delivery efficiency. Specific findings are:

1. It is feasible to guide ferromagnetic particles by means of permanent magnets to achieve clinically significant olfactory dosages. With an optimal synthesis of proper magnet layout, particle-release position, and carrier size, an olfactory delivery efficiency of $45 \%$ can be achieved.

2. The optimal particle size for magnetophoretic guidance to the olfactory region is around $15 \mu \mathrm{m}$.

3. Magnetophoretic olfactory delivery is highly sensitive to drug-release positions. Point release of drugs is essential for effective olfactory deliveries.

4. Due to the unstable nature of magnetophoresis and the quick decay of magnetic field intensity, precise control of particle motion in the human nose is still challenging.

\section{Acknowledgments}

This study was funded by Central Michigan University Innovative Research Grant P421071 and Early Career Grant P622911. Dr Jong Won Kim, James Hasbany, Alexander
Grabinski, Khaled Talaat, Zachary Firlit, and Che-Ting Ho are gratefully acknowledged for reviewing the manuscript.

\section{Disclosure}

The authors report no conflicts of interest in this work.

\section{References}

1. Mistry A, Stolnik S, Illum L. Nanoparticles for direct nose-to-brain delivery of drugs. Int J Pharm. 2009;379(1):146-157.

2. Alam S, Khan ZI, Mustafa G, et al. Development and evaluation of thymoquinone-encapsulated chitosan nanoparticles for nose-to-brain targeting: a pharmacoscintigraphic study. Int J Nanomedicine. 2012;7: 5705-5718.

3. Shi H, Kleinstreuer C, Zhang Z. Laminar airflow and nanoparticle or vapor deposition in a human nasal cavity model. J Biomech Eng. 2006; 128(5):697-706.

4. Si X, Xi J, Kim J, Zhou Y, Zhong H. Modeling of release position and ventilation effects on olfactory aerosol drug delivery. Respir Physiol Neurobiol. 2013;186(1):22-32.

5. Xi J, Longest PW. Characterization of submicrometer aerosol deposition in extrathoracic airways during nasal exhalation. Aerosol Sci Technol. 2009;43(8):808-827.

6. Türker S, Onur E, Ozer Y. Nasal route and drug delivery systems. Pharm World Sci. 2004;26(3):137-142.

7. Illum L. Nasal drug delivery: new developments and strategies. Drug Discov Today. 2002;7(23):1184-1189.

8. Dames P, Gleich B, Flemmer A, et al. Targeted delivery of magnetic aerosol droplets to the lung. Nat Nanotechnol. 2007;2(8): 495-499.

9. Shinkai M. Functional magnetic particles for medical application. J Biosci Bioeng. 2002;94(6):606-613.

10. Shapiro B. Towards dynamic control of magnetic fields to focus magnetic carriers to targets deep inside the body. J Magn Magn Mater. 2009;321(10):1594-1599.

11. Zhang L, Gu FX, Chan JM, Wang AZ, Langer RS, Farokhzad OC. Nanoparticles in medicine: therapeutic applications and developments. Clin Pharmacol Ther. 2008;83(5):761-769.

12. Pankhurst QA, Connolly J, Jones SK, Dobson J. Applications of magnetic nanoparticles in biomedicine. J Phys D Appl Phys. 2003;36(13): R167-R181.

13. Brain JD, Bloom SB, Valberg PA, Gehr P. Correlation between the behavior of magnetic iron oxide particles in the lungs of rabbits and phagocytosis. Exp Lung Res. 1984;6(2):115-131.

14. Stahlhofen W, Möller W. Behaviour of magnetic micro-particles in the human lung. Radiat Environ Biophys. 1993;32(3):221-238.

15. Jang M, Cao G. Deposition of magnetic nanoparticles suspended in the gas phase on a specific target area. Environ Sci Technol. 2006; 40(21):6730-6737.

16. Martin AR, Finlay WH. Enhanced deposition of high aspect ratio aerosols in small airway bifurcations using magnetic field alignment. J Aerosol Sci. 2008;39(8):679-690.

17. Xie Y, Zeng P, Siegel RA, Wiedmann TS, Hammer BE, Longest PW. Magnetic deposition of aerosols composed of aggregated superparamagnetic nanoparticles. Pharm Res. 2010;27(5):855-865.

18. Liang L, Zhu J, Xuan X. Three-dimensional diamagnetic particle deflection in ferrofluid microchannel flows. Biomicrofluidics. 2011;5(3): 034110 .

19. Xi J, Longest PW. Numerical predictions of submicrometer aerosol deposition in the nasal cavity using a novel drift flux approach. Int $J$ Heat Mass Transfer. 2008;51(23-24):5562-5577.

20. Zhou Y, Xi J, Simpson J, Irshad H, Cheng YS. Aerosol deposition in a nasopharyngolaryngeal replica of a 5-year-old child. Aerosol Sci Technol. 2013;47(3):275-282. 
21. Xi J, Si X, Kim J, Su G, Dong H. Modeling the pharyngeal anatomical effects on breathing resistance and aerodynamically generated sound. Med Biol Eng Comput. 2014;52(7):567-577.

22. Kim JW, Xi J, Si XA. Dynamic growth and deposition of hygroscopic aerosols in the nasal airway of a 5-year-old child. Int J Numer Method Biomed Eng. 2013;29(1):17-39.

23. Xi J, Longest PW. Transport and deposition of micro-aerosols in realistic and simplified models of the oral airway. Ann Biomed Eng. 2007; 35(4):560-581.

24. Morsi SA, Alexander AJ. An investigation of particle trajectories in two-phase flow systems. J Fluid Mech. 1972;55(2):193-208.

25. Ribeiro P, Si X. Electric and magnetic circuits. In: Beaty HW, Fink DG, editors. Standard Handbook for Electrical Engineers. 16th ed. New York: McGraw Hill; 2012:2.1-2.58.

26. Gao Y, Jian YC, Zhang LF, Huang JP. Magnetophoresis of nonmagnetic particles in ferrofluids. J Phys Chem C. 2007;111(29):10785-10791.
27. Xi J, Si X, Gaide R. Electrophoretic particle guidance significantly enhances olfactory drug delivery: a feasibility study. PLoS One. 2014;9(1):e86593.

28. Dobson J. Magnetic nanoparticles for drug delivery. Drug Dev Res. 2006;67(1):55-60.

29. Sano K, Doi M. Theory of agglomeration of ferromagnetic particles in magnetic fluids. J Phys Soc Jpn. 1983;52(8):2810-2815.

30. Satoh A, Ozaki M, Ishikawa T, Majima T. Transport coefficients and orientational distributions of rodlike particles with magnetic moment normal to the particle axis under circumstances of a simple shear flow. J Colloid Interface Sci. 2005;292(2):581-590.

31. Yellen BB, Hovorka O, Friedman G. Arranging matter by magnetic nanoparticle assemblers. Proc Natl Acad Sci U S A. 2005;102(25): 8860-8864.
International Journal of Nanomedicine

\section{Publish your work in this journal}

The International Journal of Nanomedicine is an international, peerreviewed journal focusing on the application of nanotechnology in diagnostics, therapeutics, and drug delivery systems throughout the biomedical field. This journal is indexed on PubMed Central, MedLine, CAS, SciSearch ${ }^{\circledR}$, Current Contents ${ }^{\circledR} /$ Clinical Medicine,

\section{Dovepress}

Journal Citation Reports/Science Edition, EMBase, Scopus and the Elsevier Bibliographic databases. The manuscript management system is completely online and includes a very quick and fair peer-review system, which is all easy to use. Visit http://www.dovepress.com/ testimonials.php to read real quotes from published authors. 